\title{
openheart Mechanical performance and healing patterns of the novel sirolimus-eluting bioresorbable Fantom scaffold: 6-month and 9-month follow-up by optical coherence tomography in the FANTOM II study
}

\author{
Jo Krogsgaard Simonsen, ${ }^{1}$ Emil Nielsen Holck, ${ }^{1}$ Didier Carrié, ${ }^{2}$ Norbert Frey, ${ }^{3}$ \\ Matthias Lutz, ${ }^{3}$ Joachim Weber-Albers, ${ }^{4}$ Dariusz Dudek, ${ }^{5}$ Bernard Chevalier, ${ }^{6}$ \\ Joost Daemen, ${ }^{7}$ Jouke Dijkstra, ${ }^{8}$ Camilla Fox Maule, ${ }^{1}$ Omeed Neghabat, ${ }^{1}$ \\ Jens Flensted Lassen, ${ }^{9}$ Jeffrey Anderson, ${ }^{10}$ Evald Høj Christiansen, ${ }^{1}$ \\ Alexandre Abizaid, ${ }^{11}$ Niels Ramsing Holm ${ }^{1}$
}

To cite: Simonsen JK, Holck EN, Carrié D, et al. Mechanical performance and healing patterns of the novel sirolimuseluting bioresorbable Fantom scaffold: 6-month and 9-month follow-up by optical coherence tomography in the FANTOM II study. Open Heart 2019;6:e000941. doi:10.1136/ openhrt-2018-000941

Received 29 September 2018 Revised 9 December 2018 Accepted 20 January 2019
Check for updates

(C) Author(s) (or their employer(s)) 2019. Re-use permitted under CC BY-NC. No commercial re-use. See rights and permissions. Published by BMJ.

For numbered affiliations see end of article.

Correspondence to Dr Niels Ramsing Holm ; niels. holm@clin.au.dk

\section{ABSTRACT}

Objectives We aimed to evaluate the mechanical properties and healing patterns 6 and 9 months after implantation of the sirolimus-eluting Fantom bioresorbable scaffold (BRS).

Background The Fantom BRS (Reva Medical, San Diego, USA) has differentiating properties including radiopacity, strut thickness of $125 \mu \mathrm{m}$, high expansion capacity and has demonstrated favourable mid-term clinical and angiographic outcomes.

Methods and results FANTOM II was a prospective, single arm study with implantation of the Fantom BRS in 240 patients with stable angina pectoris. Guidance by optical coherence tomography (OCT) was encouraged and was repeated at 6-month (cohort A) or 9-month follow-up (cohort B). Matched baseline and follow-up OCT recordings were available in 152 patients. In-scaffold mean lumen area in cohort $A$ was $6.8 \pm 1.7 \mathrm{~mm}^{2}$ and $5.7 \pm 1.4 \mathrm{~mm}^{2}$ at baseline and follow-up $(p<0.0001)$ and was $7.2 \pm 1.6 \mathrm{~mm}^{2}$ and $5.6 \pm 1.4 \mathrm{~mm}^{2}$ in cohort $B(p<0.0001)$. Mean scaffold area remained stable from $7.1 \pm 1.5 \mathrm{~mm}^{2}$ at baseline to $7.2 \pm 1.4 \mathrm{~mm}^{2}$ at 6 months $(\mathrm{p}=0.12)$, and from $7.4 \pm 1.5 \mathrm{~mm}^{2}$ to $7.3 \pm 1.4 \mathrm{~mm}^{2}$ at 9 months. Strut malapposition was median 0.8 (IQR $0.0 ; 3.5) \%$ and 1.8 (IQR $0.3 ; 6.0) \%$ at baseline and was 0.0 (IQR $0.0 ; 0.0) \%$ in both groups at 6-month and 9-month follow-up. Strut tissue coverage was 98.1 (IQR 95.9;99.4)\% at 6 months and 98.9 (IQR $98.3 ; 100.0) \%$ at 9 months.

Conclusions The novel Fantom BRS had favourable healing patterns at 6-month and 9-month follow-up as malapposition was effectively resolved and strut coverage was almost complete. The scaffold remained stable through follow-up with no signs of systematic late recoil.

\section{INTRODUCTION}

Fully bioresorbable scaffolds (BRS) are aimed at reducing the long-term risk associated with

\section{Key questions}

What is already known about this subject?

- Bioresorbable scaffolds (BRS) theoretically reduce the long-term risks associated with permanent drug-eluting stents (DES). However, first generation BRS had several mechanical limitations and have shown inferior clinical outcome compared to the latest generation DES. The novel Fantom BRS has demonstrated excellent mid-term clinical and angiographic outcome.

What does this study add?

- The Fantom BRS was designed to reduce limitations associated with first-generation BRS by applying a unique radiopaque backbone polymer that allows for reduced strut thickness and crossing profile while improving expansion capacity. Matched meticulous optical coherence tomography analysis revealed favourable mechanical performance and healing patterns of the scaffold after 6 and 9 months.

How might this impact on clinical practice?

- Longer term clinical follow-up in the FANTOM II study is ongoing and healing patterns are evaluated after 24 and 48 months. Studies in more complex lesion subsets are in progress and a randomised clinical outcome trial comparing the Fantom BRS with best in class permanent DES is in preparation.

permanent stent implants. Clinical trials of the first clinically available BRS have indicated increased rates of early and late scaffold failure compared with newer generation permanent drug-eluting stents (DES).${ }^{1-3}$ BRS failures have been explained by suboptimal implantation results ${ }^{45}$ but factors related to 
design and properties of the first BRS, Absorb (Abbott, USA) may have contributed negatively even in cases with optimal implantation results. Absorb BRS had thicker struts, a more dense footprint, a larger crossing profile compared with latest generation permanent DES. ${ }^{6}$ Acute recoil was larger due to the lower radial strength and the limited expansion capacity increased the risk of fractures if expanded beyond narrow limits. ${ }^{67}$

Next generation BRS were designed to address these issues for improving clinical feasibility and safety. The novel sirolimus-eluting Fantom BRS (REVA Medical, San Diego, USA) has a desaminotyrosine (DAT) based backbone polymer to improve strength and elasticity that allows for reduced strut thickness and larger expansion beyond the nominal diameter compared with Absorb BRS. The polymer of the Fantom BRS contains covalently bound iodine making the scaffold radiopaque, thus allowing for direct verification of implantation results which may further improve feasibility and safety.

The FANTOM II study aimed to investigate safety and performance of the Fantom BRS in a multicentre setting. The mid-term clinical and angiographic follow-up was reported previously and indicated an excellent safety profile. ${ }^{8}$ Here, we report the mechanical performance and healing patterns of the Fantom BRS after 6 and 9 months assessed by baseline adjusted, fully matched optical coherence tomography (OCT).

\section{METHODS}

\section{Study population}

The FANTOM II study was a prospective single-arm, multicentre study assessing clinical and angiographic outcomes after implantation of the Fantom BRS in patients $\geq 18$ years with de novo lesions and evidence of myocardial ischemia (stable or unstable angina, postinfarct angina or silent ischemia). Stenosis severity had to be $\geq 50 \%$ and $<100 \%$ in native coronary vessels with Thrombolysis in Myocardial Infarction (TIMI) flow $\geq 2$, a reference diameter between 2.5 and $3.5 \mathrm{~mm}$, and a lesion length $\leq 20 \mathrm{~mm}$ by visual assessment. Major exclusion criteria were clinical symptoms concordant with acute myocardial infarction (AMI), left main stenosis $\geq 50 \%$, ejection fraction $<40 \%$, need for more than one Fantom BRS to cover the lesion, a side branch diameter $>1.5 \mathrm{~mm}$, moderate to severe calcification and significant stenosis $\geq 50 \%$ proximal or distal to the target lesion. The study was approved by local or national medical ethics committees and health authorities as required. All patients provided written informed consent before enrolment. Clinicaltrials.gov identifier: NCT02539966.

\section{OCT study population}

Patients were eligible for the OCT substudy population if final post-PCI OCT scan and a 6-month or 9-month follow-up OCT scan were available in analysable quality.

\section{Study device}

The Fantom BRS is a sirolimus eluting scaffold with a desaminotyrosine (DAT) based backbone polymer. Iodine is covalently bound in the polymer to make it radiopaque. Strut thickness is $125 \mu \mathrm{m}$ and the in-phase sinusoidal rings are linked by three connectors. Sirolimus is incorporated into a polymeric matrix and added to the abluminal side of struts with more than $60 \%$ of the sirolimus released within 30 days. Fantom maintains structural integrity and almost full radial strength for 3-4 months followed by gradual degradation with $>60 \%$ molecular weight loss within 12 months and complete bioabsorption within 3-4 years. Deliverability is facilitated by a $1.3 \mathrm{~mm}$ crossing profile and uninterrupted device inflation was allowed. The specified postdilatation threshold was $0.5 \mathrm{~mm}$ above nominal scaffold diameter but the expansion capacity was by in vitro testing shown to exceed $1.0 \mathrm{~mm}$ above the nominal size (vendor information). The Fantom BRS was available in 2.5 $\mathrm{mm}$ and $3.0 \mathrm{~mm}$ versions during the study.

\section{Study procedure}

The treatment protocol has been published ${ }^{8}$ and was as follows (shortened): (1) predilatation of the lesion with a balloon sized 1:1 to the reference size of the vessel, (2) optional intravascular ultrasound (IVUS) or OCT imaging was recommended for measurement of reference size and lesion length, (3) selection of scaffold size according to invasive measurements or else by visual estimate, (4) implantation of the Fantom BRS to nominal pressure, (5) postdilatation with a non-compliant balloon with final size not exceeding nominal size $+0.5 \mathrm{~mm}$, (6) optional evaluation of implantation result by IVUS or OCT, (7) documentation of final result by angiography and IVUS or OCT (optional). Data describing if and how IVUS or OCT scans were used for guiding the implantation were not collected. Bail-out stenting in case of major dissection or occlusive complications at baseline was to be performed by implantation of a permanent DES.

\section{Clinical endpoints}

Endpoints reported for the OCT substudy population includes major adverse cardiac events (MACE) at 6 and 9 months; a composite of cardiac death, myocardial infarction and clinically driven target lesion revascularisation; the individual components of MACE, all-cause mortality, target vessel revascularisation and scaffold thrombosis. Endpoints were derived from the main study population and by same definitions. ${ }^{8}$

\section{OCT imaging analysis}

All OCT recordings of sufficient quality were analysed. Corresponding baseline and follow-up recordings were matched on frame level to accommodate for cardiac motion artefact and unanalysable scaffold segments (see online supplementary file 1 ). Analysis was performed with a sample rate of 0.5 or $0.6 \mathrm{~mm}$ to accommodate for differences in pullback speed. The highly back scattering Fantom BRS required an adaptable analysis method implying a 


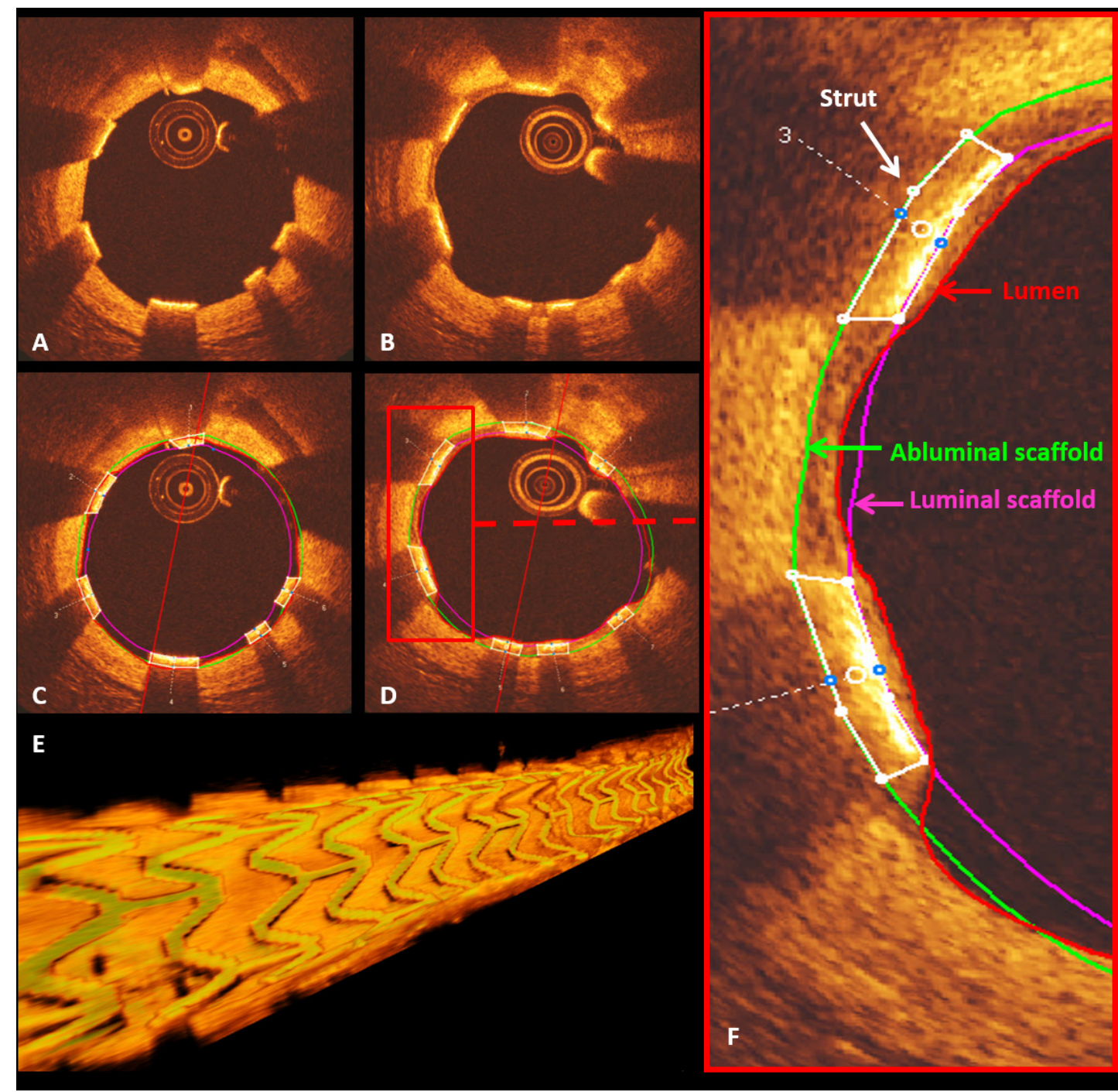

Figure 1 OCT appearance and analysis of the Fantom scaffold. (1) OCT image acquisitions showing the unique appearance of the Fantom BRS by OCT scan at 6 months. The Fantom BRS was easily distinguished at baseline $(A, C)$ with a highly backscattering luminal surface. Due to high attenuation, the abluminal strut surface was not identifiable and analysis was performed by applying a customised box delineating the strut with a fixed thickness (Type-R) (C,D,F). At the time of followup, the Fantom BRS remained clearly discernible despite tissue coverage and progressing strut degradation (B,D). Owing to the unique appearance of the Fantom BRS by OCT, direct 3D OCT rendering was feasible yielding a well-defined scaffold reconstruction without the need for strut segmentation (E). Panel F shows follow-up analysis with lumen contour (red line), strut box delineation (white boxes), luminal and abluminal scaffold contours (pink and green lines). BRS, bioresorbable scaffolds; OCT, optical coherence tomography.

fixed strut thickness of $125 \mu \mathrm{m}$ (figure 1). A box was fitted to each strut in analysed frames allowing for construction of abluminal scaffold contours and detection of possible malapposition as distance between the abluminal side of the artificial strut box and the lumen contour. OCT endpoints were mean lumen area, minimal lumen area, mean scaffold area, minimal scaffold area, mean neointimal hyperplasia area, mean neointimal thickness, mean extra-stent lumen area, strut malapposition, strut tissue coverage, scaffold fracture, discontinued struts and in-scaffold thrombus. Strut tissue coverage was analysed on strut level and defined as identifiable tissue fully covering the luminal strut surface. Malapposed struts were analysed on strut level and defined as struts separated from the luminal vessel surface detected as a clear separation of the fitted box and the lumen contour abluminal to the strut. Analysis for fractured struts at baseline and discontinued struts at follow-up was performed at patient level by 3D OCT detection. Strut fracture and discontinuity were defined as overlapping struts in non-overlapping segments, single ended strut protrusion of struts into lumen or any abrupt strut discontinuity. Thrombus was analysed on patient level and defined as any degree of thrombus-material on or attached to a strut observed in at least two consecutive frames. Derived endpoints at follow-up were lumen area late loss (baseline minimal in-scaffold lumen area - follow-up minimal in-scaffold lumen area) and mean lumen enlargement (follow-up mean in-scaffold lumen area - baseline 
mean in-scaffold lumen area). OCT analysis was performed by the Interventional Coronary Imaging Core Laboratory (Aarhus University Hospital, Skejby, Denmark) using the QCU-CMS software (Leiden University Medical Center, The Netherlands) and QangioOCT RE (Medis medical imaging, The Netherlands).

\section{Statistical analysis}

Categorical variables are reported as mean percentages with SD. Continuous variables with a normal distribution are reported as mean with SD and mean differences with $95 \%$ CI. Data were analysed on patient level equalling lesion level equalling single study stent level as all study patients only had one stent implanted and in one lesion. Strut and frame level data were analysed in two steps: first on per patient level by simple average and in a second step per patient with an over all mean of all observations. Plots of individual patient's patient level data are provided for main OCT endpoint measures. Continuous variables not following normal distribution are reported as medians with interquartiles. Testing of matched baseline and follow-up results on patient level was performed with simple paired t-test for normal distributed data and non-parametric Wilcoxon signed rank test for data not following a normal distribution.

\section{RESULTS}

\section{Patient population}

A total of 240 patients were enrolled in the FANTOM II study. The first 117 patients were enrolled in cohort A with OCT acquisition performed in 89 patients at baseline and in 81 patients at follow-up. The last 123 patients were enrolled in cohort B with OCT performed in 98 patients at baseline and in 94 patients at follow-up (online supplementary figure 2). Matched analysis of corresponding baseline and follow-up OCT recordings was possible for 73 patients in cohort $\mathrm{A}$ and 79 patients in cohort B (matched population, figure 2). Mean follow-up time of the matched cohort was 194 (15) days in cohort A and 287 (38) days in cohort B. Patient characteristics are presented in table 1 and baseline procedural outcomes are presented in table 2 .

\section{Clinical outcomes}

Clinical events for patients with matched OCT analysis: at 6-month follow-up in cohort A, one patient underwent non-clinically driven target lesion revascularization (TLR). Cohort B: Four patients in the matched OCT cohort who underwent TLR and OCT from all these procedures were included in the matched analysis. In patients excluded from the matched OCT cohort due to missing follow-up OCT one death, one myocardial infarction and three TLR were reported. Flowchart for patients not included in the matched analysis is shown in online supplementary file 1 .

\section{OCT imaging results}

Qualitative assessment of OCT signature

The Fantom BRS was easily distinguished in both baseline and 6-month and 9-month follow-up OCT recordings exhibiting a highly backscattering luminal surface and an abluminal strut border not discernible due to high attenuation. Visibility of the Fantom BRS in 3D OCT reconstructions by proprietary online tools without the

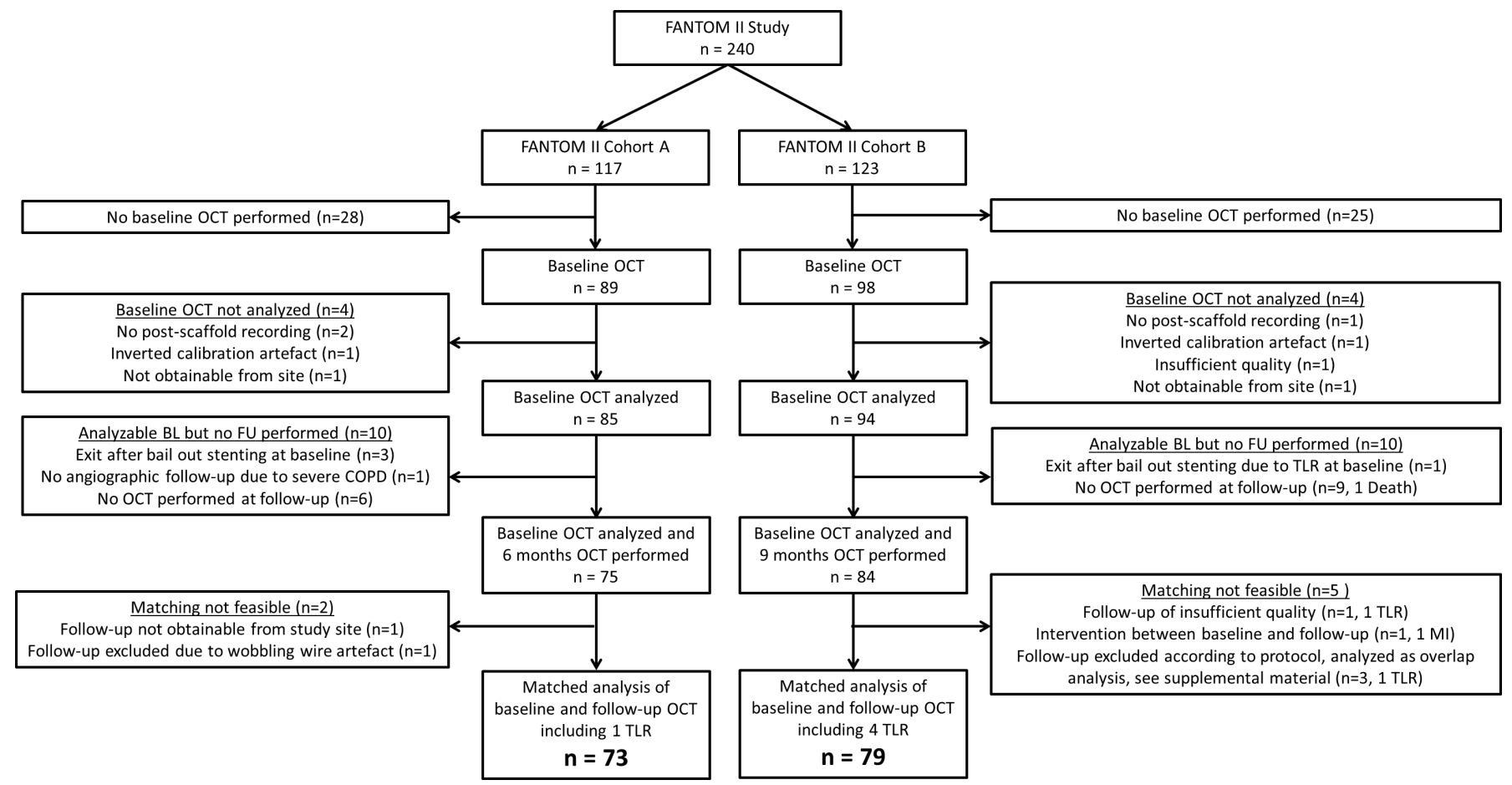

Figure 2 Flowchart of the matched patient population in the FANTOM II Study with a total of 240 patients enrolled at baseline. Frame level matched analysis of baseline and follow-up recordings were available in 73 patients in cohort $A$ and 79 patients in cohort B. $n=$ patients. OCT, optical coherence tomography. 


\begin{tabular}{|c|c|c|}
\hline Baseline data & Cohort A $(n=73)$ & Cohort B $(n=79)$ \\
\hline Age (years) & $62.7 \pm 9.0$ & $62.2 \pm 10.6$ \\
\hline Male gender & $52(71.2 \%)$ & $64(81.0 \%)$ \\
\hline Smoking history & $39(53.4 \%)$ & $51(64.6 \%)$ \\
\hline $\begin{array}{l}\text { Current smoker (within the } \\
\text { past } 2 \text { weeks) }\end{array}$ & $15(20.5 \%)$ & $16(20.3 \%)$ \\
\hline Diabetes & $12(16.4 \%)$ & 22 (27.9\%) \\
\hline Hypertension & $53(72.6 \%)$ & $54(68.4 \%)$ \\
\hline Hypercholesterolemia & $50(68.5 \%)$ & $55(69.6 \%)$ \\
\hline Family history of CAD & $27(37.0 \%)$ & $28(35.4 \%)$ \\
\hline Prior MI & $20(27.4 \%)$ & $21(26.6 \%)$ \\
\hline Prior PCl & $28(38.4 \%)$ & $39(49.4 \%)$ \\
\hline
\end{tabular}

Patient characteristics for the 73 patients in cohort $A$ and the 79 patients in cohort B included in the matched OCT analysis. Values are $\mathrm{n}(\%)$ or mean \pm SD.

$\mathrm{CAD}$, coronary artery disease; $\mathrm{MI}$, myocardial infarction; OCT, optical coherence tomography; $\mathrm{PCl}$, percutaneous coronary intervention.

need for strut detection and segmentation was confirmed (figure 1).

\section{Quantitative 0CT analysis}

Matched analysis of OCT recordings in cohort A (73 patients) had a total strut count of 40929 and in cohort B (79 patients) the strut count was 46 649. Results are presented in table 3. Data analysis revealed a small decrease in mean lumen area from $6.8(1.7) \mathrm{mm}^{2}$ to 5.7 (1.4) $\mathrm{mm}^{2}$ in cohort $\mathrm{A}(\mathrm{p}<0.0001)$ and from $7.2(1.6)$ $\mathrm{mm}^{2}$ to $5.6(1.4) \mathrm{mm}^{2}$ in cohort $\mathrm{B}(\mathrm{p}<0.0001)$. A similar decrease in minimal lumen area was observed in cohort $\mathrm{A}$ from $5.3(1.4) \mathrm{mm}^{2}$ to $4.3(1.3) \mathrm{mm}^{2}$ and in cohort B from $5.7(1.4) \mathrm{mm}^{2}$ to $4.1(1.3) \mathrm{mm}^{2}$. There was no evidence of scaffold area reduction from post-PCI (percutaneous coronary intervention) to 6-month or 9-month follow-up as mean scaffold area remained stable from $7.1(1.5) \mathrm{mm}^{2}$ to $7.2(1.4) \mathrm{mm}^{2}(\mathrm{p}=0.12)$ in cohort A and from $7.4(1.5)$ $\mathrm{mm}^{2}$ to $7.3(1.4) \mathrm{mm}^{2}$ in cohort $\mathrm{B}(\mathrm{p}=0.13)$. Minimal scaffold area was unchanged in cohort A from $5.9(1.3) \mathrm{mm}^{2}$ to $6.0(1.3) \mathrm{mm}^{2}(\mathrm{p}=0.08)$ and in cohort $\mathrm{B}$ from 6.1 (1.4) $\mathrm{mm}^{2}$ to $6.1(1.3) \mathrm{mm}^{2}(\mathrm{p}=0.40)$. Strut level malapposition counts were median $0.8(0.0 ; 3.5) \%$ at baseline in cohorts $\mathrm{A}$ and $1.8(0.3 ; 6.0) \%$ in cohort $\mathrm{B}$. In both cohort $\mathrm{A}$ and cohort B, malapposition was almost resolved at follow-up with a median of $0.0 \quad(0.0 ; 0.0) \%$ (figure 3). Tissue coverage of struts was complete for $98.1(95.9 ; 99.4) \%$ of struts at 6-month follow-up and $98.9(98.3 ; 100.0) \%$ at 9 months (figure 3). Strut fracture at baseline was found in seven patients, three in scaffolds exposed to excessive expansion and four had subtle discontinuity of a single ring or connector without obvious explanation. Strut discontinuity at follow-up was seen in four patients at 6 months including two cases with fracture at baseline. At 9 months, strut discontinuity was seen in 16 patients of
Table 2 Baseline lesion and procedural outcomes

\begin{tabular}{|c|c|c|}
\hline Baseline QCA & $\begin{array}{l}\text { Cohort A } \\
\left(\mathrm{n}=72^{*}\right)\end{array}$ & $\begin{array}{l}\text { Cohort B } \\
(\mathrm{n}=79)\end{array}$ \\
\hline \multicolumn{3}{|l|}{ Target vessel } \\
\hline $\begin{array}{l}\text { Left Anterior Decending artery } \\
\text { (LAD) }\end{array}$ & $37(51.4 \%)$ & $41(51.9 \%)$ \\
\hline Circumflex artery $(\mathrm{Cx})$ & $22(30.6 \%)$ & $20(25.3 \%)$ \\
\hline Right Coronary Artery (RCA) & $13(18.1 \%)$ & $18(22.8 \%)$ \\
\hline \multicolumn{3}{|l|}{ ACC/AHA lesion classification } \\
\hline Type A & $21(29.2 \%)$ & $9(11.4 \%)$ \\
\hline Type B1 & $26(36.1 \%)$ & $44(55.7 \%)$ \\
\hline Type B2 & $25(34.7 \%)$ & $22(28.8 \%)$ \\
\hline Type C & $0(0.0 \%)$ & $4(5.1 \%)$ \\
\hline Reference vessel diameter (mm) & $2.67 \pm 0.34$ & $2.74 \pm 0.34$ \\
\hline Lesion length $(\mathrm{mm})$ & $10.8 \pm 3.5$ & $12.2 \pm 4.3$ \\
\hline Procedural data & $\underline{\mathrm{n}=73}$ & $\underline{\mathrm{n}=79}$ \\
\hline \multicolumn{3}{|l|}{ Preprocedure antiplatelets } \\
\hline Aspirin & $73(100.0 \%)$ & $79(100.0 \%)$ \\
\hline Ticagrelor or clopidogrel & $72(98.6 \%)$ & $79(100.0 \%)$ \\
\hline Other antithrombotic agent & $1(1.4 \%)$ & $0(0.0 \%)$ \\
\hline Nominal scaffold diameter (mm) & $2.88 \pm 0.21$ & $2.89 \pm 0.21$ \\
\hline Nominal scaffold length (mm) & $18.1 \pm 0.70$ & $19.0 \pm 2.20$ \\
\hline Predilatation & $73(100.0 \%)$ & $79(100.0 \%)$ \\
\hline Postdilatation & $62(84.9 \%)$ & $70(88.6 \%)$ \\
\hline $\begin{array}{l}\text { Maximal postdilatation pressure } \\
\text { (atm) }\end{array}$ & $17 \pm 4$ & $17 \pm 5$ \\
\hline $\begin{array}{l}\text { Maximal postdilatation balloon } \\
\text { diameter }(\mathrm{mm})\end{array}$ & $3.15 \pm 0.36$ & $3.26 \pm 0.34$ \\
\hline \multicolumn{3}{|l|}{ Antiplatelets at follow-up } \\
\hline Aspirin & $73(100.0 \%)$ & $78(98.7 \%)$ \\
\hline Ticagrelor or clopidogrel & $73(100.0 \%)$ & $79(100.0 \%)$ \\
\hline
\end{tabular}

Baseline procedural outcome for patients included in the matched analysis. Values are $\mathrm{n}(\%)$ or mean \pm SD.

${ }^{*}$ One preprocedure image was not available for analysis.

QCA, quantitative coronary arteriography.

whom five were persisting discontinuities and 11 were acquired. Importantly, all but one case with fracture or late discontinuity had fully apposed struts in the affected segments at follow-up and none had clinical events. Results of unmatched analysis are presented in online supplementary table 1.

\section{DISCUSSION}

The favourable main 6-month and 9-month OCT results confirm the previously reported clinical and angiographic data. The OCT results indicated that the intended performance and healing patterns of the novel Fantom BRS achieved: (1) a small but expected decrease in mean and minimal lumen area; (2) stable minimal scaffold area at follow-up; (3) acute malapposition was effectively resolved; (4) no tendency to late vessel wall 


\section{Table 3 Matched OCT results}

\begin{tabular}{|c|c|c|c|c|}
\hline Matched OCT results & Baseline & Follow-up & Difference & $P$ value \\
\hline \multicolumn{5}{|l|}{ Mean lumen area $\left(\mathrm{mm}^{2}\right)$} \\
\hline 6 months & $6.8(1.7)$ & $5.7(1.4)$ & $1.1(-1.3 ;-0.9)$ & $<0.0001$ \\
\hline 9 months & $7.2(1.6)$ & $5.6(1.4)$ & $1.6(-1.7 ;-1.4)$ & $<0.0001$ \\
\hline \multicolumn{5}{|l|}{ Minimal lumen area $\left(\mathrm{mm}^{2}\right)$} \\
\hline 6 months & $5.3(1.4)$ & $4.3(1.3)$ & $1.0(-1.3 ;-0.7)$ & $<0.0001$ \\
\hline 9 months & $5.7(1.4)$ & $4.1(1.3)$ & $1.6(-1.9 ;-1.4)$ & $<0.0001$ \\
\hline \multicolumn{5}{|l|}{ Mean scaffold area $\left(\mathrm{mm}^{2}\right)$} \\
\hline 6 months & $7.1(1.5)$ & $7.2(1.4)$ & $0.1(-0.02 ; 0.24)$ & 0.12 \\
\hline 9 months & $7.4(1.5)$ & $7.3(1.4)$ & $-0.1(-0.19 ; 0.02)$ & 0.12 \\
\hline 9 months & $7.4(1.5)$ & $7.3(1.4)$ & $-0.1(-0.19 ; 0.02)$ & 0.13 \\
\hline \multicolumn{5}{|l|}{ Minimal scaffold area $\left(\mathrm{mm}^{2}\right)$} \\
\hline 6 months & $5.9(1.3)$ & $6.0(1.3)$ & $0.1(-0.02 ; 0.25)$ & 0.08 \\
\hline 9 months & $6.1(1.4)$ & $6.1(1.3)$ & $-0.1(-0.24 ; 0.10)$ & 0.40 \\
\hline \multicolumn{5}{|c|}{ Mean extra scaffold lumen area $\left(\mathrm{mm}^{2}\right)^{\star}$} \\
\hline 6 months & $0.05(0.02 ; 0.13)$ & $0.00(0.00 ; 0.02)$ & $0.04(-0.11 ;-0.02)$ & $<0.0001$ \\
\hline 9 months & $0.09(0.04 ; 0.19)$ & $0.00(0.00 ; 0.02)$ & $0.08(-1.16 ;-0.03)$ & $<0.0001$ \\
\hline \multicolumn{5}{|l|}{ Mean neointimal area $\left(\mathrm{mm}^{2}\right)^{*}$} \\
\hline 6 months & & $1.2(1.0 ; 1.4)$ & & \\
\hline 9 months & & $1.4(1.1 ; 1.7)$ & & \\
\hline \multicolumn{5}{|l|}{ Mean neointimal thickness $(\mathrm{mm})^{*}$} \\
\hline 6 months & & $0.057(0.040 ; 0.077)$ & & \\
\hline 9 months & & $0.072(0.056 ; 0.101)$ & & \\
\hline \multicolumn{5}{|l|}{ Total analysed struts $(\mathrm{N})$} \\
\hline 6 months & 20696 & 20233 & & \\
\hline 9 months & 23775 & 22875 & & \\
\hline \multicolumn{5}{|c|}{ Mean strut count per analysis N (SD) } \\
\hline 6 months & $283(56)$ & $277(57)$ & & \\
\hline 9 months & $301(62)$ & $290(62)$ & & \\
\hline \multicolumn{5}{|l|}{ Malapposed struts (\%)† } \\
\hline 6 months & $0.8(0.0 ; 3.5)$ & $0.0(0.0 ; 0.0)$ & $-0.7(-3.5 ; 0)$ & $<0.0001$ \\
\hline 9 months & $1.8(0.3 ; 6.0)$ & $0.0(0.0 ; 0.0)$ & $-1.5(-5.1 ;-0.3)$ & $<0.0001$ \\
\hline \multicolumn{5}{|l|}{ Covered struts (\%)† } \\
\hline 6 months & & $98.1(95.9 ; 99.4)$ & & \\
\hline 9 months & & $98.9(98.3 ; 100.0)$ & & \\
\hline Discontinued struts $\mathrm{N}(\%) \ddagger$ & $2(2.7)$ & $4(5.5)$ & $2.7(-2.4 ; 7.9)$ & 0.16 \\
\hline 6 months & $5(6.3)$ & $16(20.3)$ & $13.9(2.9 ; 24.9)$ & 0.01 \\
\hline 9 months & $5(6.3)$ & $16(20.3)$ & $13.9(2.9 ; 24.9)$ & 0.01 \\
\hline \multicolumn{5}{|l|}{ Thrombus N (\%)‡ } \\
\hline 6 months & $48(65.8)$ & $2(2.7)$ & & \\
\hline 9 months & $43(54.4)$ & $2(2.5)$ & & \\
\hline
\end{tabular}

Matched data from the 73 patients in Cohort A and 79 patients in Cohort B. Results are mean (SD).

*Median (interquartile).

†Analysed on strut level, reported as median (IQR).

$\ddagger$ Analysed on patient level.

OCT, optical coherence tomography. 


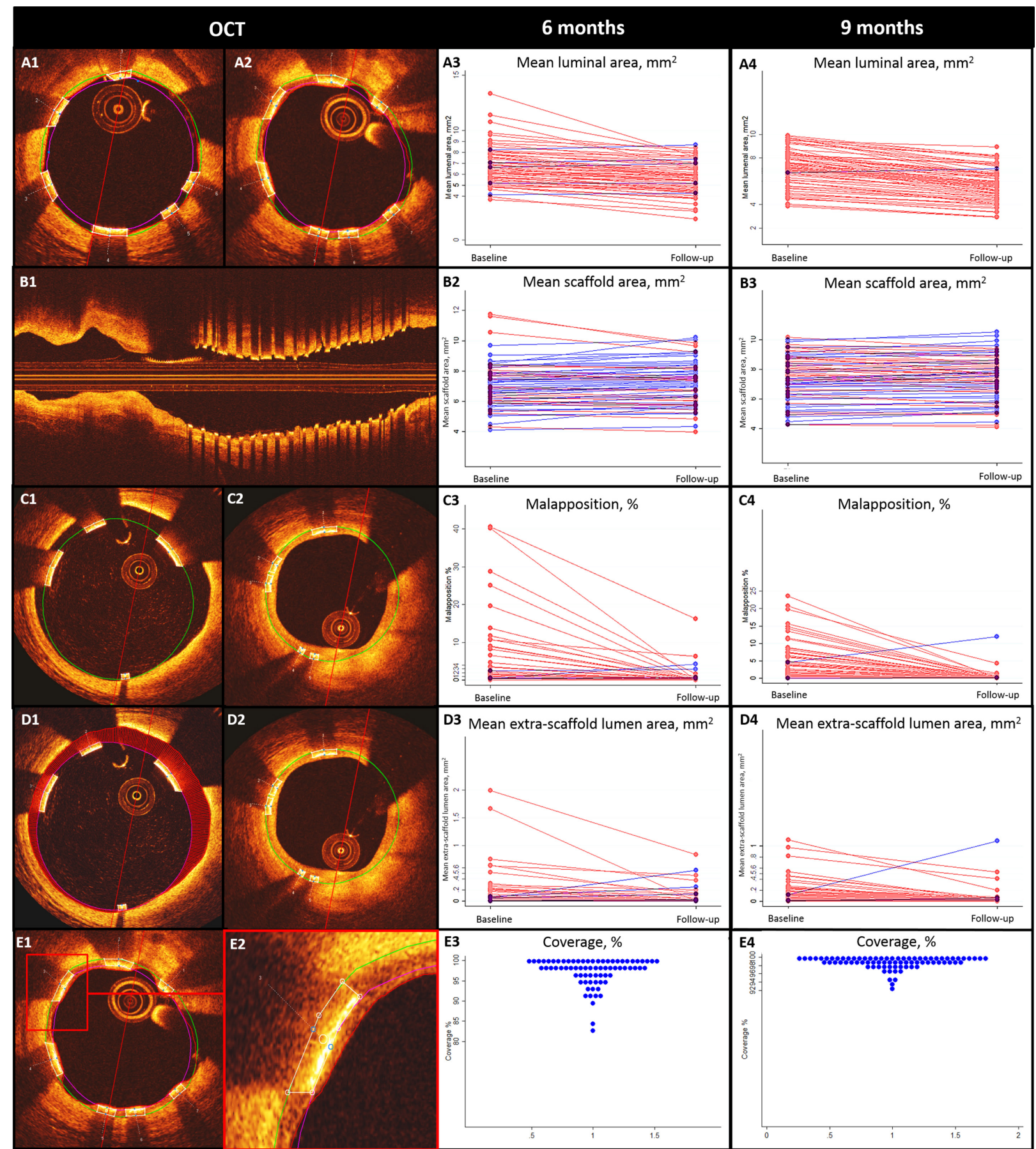

Figure 3 Healing patterns assessed by OCT. Matched OCT analysis of baseline $(\mathrm{A} 1, \mathrm{~B} 1)$ and follow-up (A2) recordings revealed a slight decrease in mean luminal area after 6 and 9 months as illustrated by the interconnected mean baseline and follow-up values $(\mathrm{A} 3, \mathrm{~A} 4)$ while no evidence of scaffold area reduction was observed $(\mathrm{B} 2, \mathrm{~B} 3)$. An excellent healing response to acute malapposition (C1) with almost completely resolved malapposition at the time of follow-up (C2) even in the few cases of major malapposition at baseline (C3,C4). Mean extra-scaffold lumen area at baseline (D1) had also diminished at the time of follow-up (D2) even in the few cases of major extra-scaffold lumen area at baseline (D3,D4). A balanced neointimal response was observed with a thin layer of tissue covering the struts $(E 1, E 2)$ in the majority of cases agreeing with the high level of strut coverage seen in this study (E3,E4). OCT, optical coherence tomography.

detachment, formation of evaginations or aneurysms and (5) strut coverage was almost complete at the 6-month and 9-month follow-up.

Introduction of the Absorb BRS was a milestone in treatment of obstructive coronary artery disease. The positive initial safety reports, ${ }^{9}$ the restoration of the vasomotor function, ${ }^{10}$ the late lumen enlargement ${ }^{11}$ and the full resorption documented after 5 years ${ }^{12}$ were encouraging for the technology. However, recent reports on early luminal scaffold dismantling, ${ }^{4}$ increased rates of scaffold thrombosis ${ }^{12}$ and a late increase in target lesion failures in comparison with permanent $\mathrm{DES}^{23}$ indicates 
that limitations of first generation BRS affect outcomes negatively. Factors related to the BRS design potentially contributing to scaffold failure include the bulky struts, ${ }^{13} 14$ the increased scaffold footprint, ${ }^{15}$ the limited expansion capacity, ${ }^{67}$ limited radial strength, ${ }^{6}{ }^{16}$ degradation and embolisation of struts in the lumen due to persisting malapposition or late wall detachment of the scaffold ${ }^{17}$ and the poor guidance by angiography. ${ }^{18}$

In comparison to the Absorb BRS, the Fantom BRS was designed with thinner struts to facilitate healing and reduce flow disturbance. At the same time, the Fantom polymer allowed for maintaining radial strength, increased expansion capacity and was radiopaque to improve detection of under expansion and scaffold position thereby reducing the risk of geographical miss and aiding serial implantation of scaffolds.

In the FANTOM II study, we documented that malapposition was effectively resolved and coverage was almost complete at 6 and 9 months. Even in cases with substantial acute malapposition due to underestimation of the reference size by the treating physician, malapposition was effectively reduced. Still, the healing response was balanced as indicated by the very few cases with a more pronounced reduction in minimal lumen area at follow-up. Adverse healing response with development of evaginations and aneurysms was a concern with first generation $\mathrm{DES}^{1920}$ and similar healing patterns was reported in cases with Absorb BRS. ${ }^{21}$ With the Fantom BRS, we found a substantial reduction in extra stent lumen indicating a favourable healing response with only two of 152 cases having isolated bulging vessel and no cases had generalised patterns of evaginations.

Despite the reduced strut thickness and substantial reduction in molecular weight at 6 and 9 months, the Fantom BRS maintained stable mean and minimal scaffold area at follow-up and with no late vessel wall detachment. If this important resistance to late recoil continues at longer-term follow-up, it may indicate an additional differentiating property of the Fantom BRS. The Fantom BRS platform allows for expansion beyond the nominal size by at least $0.7 \mathrm{~mm}$ and safe in vitro expansion up to $1.0 \mathrm{~mm}$ has been reported by the vendor. By highly sensitive 3D OCT analysis, we identified a total of $7 / 152$ scaffolds with fracture at baseline. Three were caused by excessive expansion in attempts to correct malapposition due to severe underestimation of the true vessel size, and four had no evident explanation but was isolated to a single strut or ring discontinuity. None of the patients with fracture at baseline had clinical events. Similar findings related to overexpansion were reported in ABSORB cohort B which by a less sensitive method revealed acute disruption during baseline procedure in 2 out of 51 patients $(3.9 \%)$ possibly related to over expansion of the scaffold. ${ }^{4}$ These findings emphasise that despite increased inflation thresholds, meticulous sizing is a prerequisite also with the next generation BRS. The late acquired strut discontinuity seen in $2.7 \%$ of cases after 6 months compared with 12\% in Absorb
$\mathrm{BRS}^{4}$ could indicate better structural integrity of Fantom BRS at this time point. Some discontinuity at follow-up is expected due to resorption and should only be seen as problematic if causing lumen reduction or protrusion of struts into the lumen. Thrombus elements on struts were found in some patients during implantation but not at follow-up. This finding did not translate to any clinical scaffold thrombosis and, based on the non-comparative material it was not possible to determine if the level was higher than with regular permanent DES. Still, with the present level of evidence, it is encouraged to adhere to the mandatory preloading with aspirin and clopidogrel or ticagrelor and to maintain dual anti platelet therapy for 12 months after implantation.

The FANTOM II study findings indicate that the differentiating design features between the Fantom BRS and the Absorb BRS may prove of importance in addressing safety concerns raised with first generation BRS. Longer term follow-up, studies in more complex lesion subsets and adequately powered randomised trials are necessary to evaluate if improved structural properties and healing patterns of the Fantom BRS translates in to clinical outcomes allowing for routine clinical use.

\section{Limitations}

The FANTOM II study selection criteria only allowed enrolment of patients with rather simple lesions limiting the assessment of safety and efficacy after implantation in complex lesions. Despite the favourable results, the single arm design did not allow for a direct comparison of results to established treatments. As with other imaging follow-up studies, there could be a bias towards the healthy survivors as not all patients were evaluated by OCT at baseline and follow-up. By matching the recordings, 27 patients with analysable baseline OCT but no available follow-up OCT were excluded from analysis. Still, unmatched OCT results were remarkably close to the matched OCT results and the consequences of matching may be considered minimal and outweighed by the advantages (online supplementary table 1).

\section{Conclusion}

Treatment of coronary stenosis by implantation of the Fantom BRS resulted in favourable 6-month and 9-month healing patterns as assessed by OCT. Scaffold area remained stable, the healing response caused a limited lumen reduction, malapposition was effectively resolved and strut coverage was almost complete at the 6-month and 9-month time points.

\section{Author affiliations \\ ${ }^{1}$ Department of Cardiology, Aarhus University Hospital, Skejby, Aarhus, Denmark ${ }^{2}$ Department of Cardiology, University Hospital Rangueil, Toulouse, France ${ }^{3}$ Department of Cardiology and Angiology, University Medical Center Schleswig- Holstein, Kiel, Germany \\ ${ }^{4}$ St. Johannes-Hospital, Dortmund, Germany \\ ${ }^{5}$ Institute of Cardiology, Jagiellonian University Medical College, University Hospital, Krakow, Poland \\ ${ }^{6}$ Department of Cardiology, Institut Cardiovasculaire Paris Sud, Massy, France}


${ }^{7}$ Department of Cardiology, Erasmus Universiteit Rotterdam, Rotterdam, Netherlands

${ }^{8}$ Division of Image Processing, Leiden University Medical Center, Leiden, Netherlands

${ }^{9}$ The Heart Center, Rigshospitalet, Copenhagen, Denmark

${ }^{10}$ REVA Medical Inc, San Diego, USA

${ }^{11}$ Instituto de Cardiologia Dante Pazzanese, Sao Paulo, Brazil

Contributors JS: study design, OCT analysis, data analysis, drafting manuscript, interpretation; direct access to all data. EH: study design, study management, OCT analysis, interpretation; critical revision; direct access to all data. DC, NF, JW-A DD, BC, JDa: patient enrolment, interpretation, critical revisions. ML: design, patient enrolment, interpretation, critical revisions. JDi: analysis design, OCT analysis, interpretation, critical revision. CFM: OCT analysis, interpretation, critical revision. ON: analysis design, OCT analysis, interpretation, critical revision. JL: analysis design, national coordinator, OCT analysis, interpretation, critical revision. JA: study design, study setup, study management, critical revision. EHC: study design, patient enrolment, interpretation, critical revision. AA: study design, patient enrolment, interpretation, critical revision. NRH: study design, OCT analysis, data analysis, interpretation, drafting of manuscript; direct access to all data; final responsibility.

Funding This article was funded by REVA Medical Inc., San Diego, USA.

Competing interests JKS received travel grants from St. Jude Medical, and institutional research grant from Reva Medical. AA received consulting fees and research grants from Reva Medical. NRH has received institutional research grants from Abbott, Boston Scientific, Reva Medical and Elixir, and speaker fees from Abbott, Reva Medical and Biotronik. EHC has received institutional research grant from Abbott, Biotronik, OrbusNiech, Reva Medical and Elixir, and speaker fees from Abbott and Terumo. Allother authors report no disclosures.

Patient consent for publication Obtained.

Ethics approval The study was approved by national health authorities and local or national medical ethics committees as appropriate

Provenance and peer review Not commissioned; externally peer reviewed.

Data sharing statement Undecided.

Open access This is an open access article distributed in accordance with the Creative Commons Attribution Non Commercial (CC BY-NC 4.0) license, which permits others to distribute, remix, adapt, build upon this work non-commercially, and license their derivative works on different terms, provided the original work is properly cited, appropriate credit is given, any changes made indicated, and the use is non-commercial. See: http://creativecommons.org/licenses/by-nc/4.0/.

\section{REFERENCES}

1. Chevalier B, Onuma Y, van Boven AJ, et al. Randomised comparison of a bioresorbable everolimus-eluting scaffold with a metallic everolimus-eluting stent for ischaemic heart disease caused by de novo native coronary artery lesions: the 2-year clinical outcomes of the absorb II trial. Eurolntervention 2016;12:1102-7.

2. Onuma $\mathrm{Y}$, Sotomi $\mathrm{Y}$, Shiomi H, et al. Two-year clinical, angiographic, and serial optical coherence tomographic follow-up after implantation of an everolimus-eluting bioresorbable scaffold and an everolimus-eluting metallic stent: insights from the randomised absorb Japan trial. Eurolntervention 2016;12:1090-101.

3. Serruys PW, Chevalier B, Sotomi Y, et al. Comparison of an everolimus-eluting bioresorbable scaffold with an everolimus-eluting metallic stent for the treatment of coronary artery stenosis (absorb II): a 3 year, randomised, controlled, single-blind, multicentre clinical trial. The Lancet 2016;388:2479-91.

4. Onuma Y, Serruys PW, Muramatsu T, et al. Incidence and imaging outcomes of acute scaffold disruption and late structural discontinuity after implantation of the absorb everolimus-eluting fully bioresorbable vascular scaffold: optical coherence tomography assessment in the absorb cohort B trial (a clinical evaluation of the bioabsorbable everolimus Eluting coronary stent system in the treatment of patients with de novo native coronary artery lesions). JACC Cardiovasc Interv 2014;7:1400-11.

5. Ishibashi $Y$, Nakatani S, Sotomi Y, et al. Relation Between Bioresorbable Scaffold Sizing Using QCA-Dmax and Clinical Outcomes at 1 Year in 1,232 Patients From 3 Study Cohorts (ABSORB Cohort B, ABSORB EXTEND, and ABSORB II). JACC Cardiovasc Interv 2015;8:1715-26.

6. Ormiston JA, Webber B, Ubod B, et al. An independent bench comparison of two bioresorbable drug-eluting coronary scaffolds (absorb and DESolve) with a durable metallic drug-eluting stent (ML8/Xpedition). Eurolntervention 2015;11:60-7.

7. Foin N, Lee R, Mattesini A, et al. Bioabsorbable vascular scaffold overexpansion: insights from in vitro post-expansion experiments. Eurolntervention 2016;11:1389-99.

8. Abizaid A, Carrié D, Frey N, et al. 6-month clinical and angiographic outcomes of a novel radiopaque sirolimus-eluting Bioresorbable vascular scaffold. JACC Cardiovasc Interv 2017;10:1832-8.

9. Serruys PW, Onuma Y, Ormiston JA, et al. Evaluation of the second generation of a Bioresorbable everolimus drug-eluting vascular scaffold for treatment of de novo coronary artery stenosis. Circulation 2010;122:2301-12.

10. Gogas BD, Benham JJ, Hsu S, et al. Vasomotor function comparative assessment at 1 and 2 years following implantation of the absorb everolimus-eluting Bioresorbable vascular scaffold and the Xience $V$ everolimus-eluting metallic stent in porcine coronary arteries: insights from in vivo angiography, ex vivo assessment, and gene analysis at the Stented/Scaffolded segments and the proximal and distal edges. JACC Cardiovasc Interv 2016;9:728-41.

11. Simsek $C$, Karanasos A, Magro M, et al. Long-term invasive followup of the everolimus-eluting bioresorbable vascular scaffold: fiveyear results of multiple invasive imaging modalities. Eurolntervention 2016;11:996-1003.

12. Serruys PW, Ormiston J, van Geuns RJ, et al. A Polylactide Bioresorbable scaffold Eluting everolimus for treatment of coronary stenosis: 5-year follow-up. J Am Coll Cardiol 2016;67:766-76.

13. Bourantas CV, Papafaklis MI, Kotsia A, et al. Effect of the endothelial shear stress patterns on neointimal proliferation following drug-eluting bioresorbable vascular scaffold implantation: an optical coherence tomography study. JACC Cardiovasc Interv 2014; 7:315-24.

14. Pache J, Kastrati A, Mehilli J, et al. Intracoronary stenting and angiographic results: strut thickness effect on restenosis outcome (ISAR-STEREO-2) trial. J Am Coll Cardiol 2003;41:1283-8.

15. Puricel S, Cuculi F, Weissner M, et al. Bioresorbable coronary scaffold thrombosis: multicenter comprehensive analysis of clinical presentation, mechanisms, and predictors. J Am Coll Cardiol 2016;67:921-31.

16. Foin $N$, Lee R, Bourantas $C$, et al. Bioresorbable vascular scaffold radial expansion and conformation compared to a metallic platform: insights from in vitro expansion in a coronary artery lesion model. Eurolntervention 2016;12:834-44.

17. Capranzano P, Francaviglia B, Capodanno D, et al. Embolization of fractured Bioresorbable scaffold Struts: insights from 2- and 3-dimensional optical coherence tomography. JACC Cardiovasc Interv 2016;9:e37-8.

18. Subban V, Sengottuvelu G, Uthayakumaran K, et al. Prevalence of parameters of suboptimal scaffold deployment following angiographic guided bioresorbable vascular scaffold implantation in real world practice - an optical coherence tomography analysis. Int $J$ Cardiol 2016;220:32-42

19. Joner M, Finn AV, Farb A, et al. Pathology of drug-eluting stents in humans: delayed healing and late thrombotic risk. J Am Coll Cardiol 2006;48:193-202.

20. Virmani R, Guagliumi G, Farb A, et al. Localized hypersensitivity and late coronary thrombosis secondary to a sirolimus-eluting stent. Circulation 2004;109:701-5.

21. Gori T, Jansen T, Weissner M, et al. Coronary evaginations and peri-scaffold aneurysms following implantation of bioresorbable scaffolds: incidence, outcome, and optical coherence tomography analysis of possible mechanisms. Eur Heart J 2016;37:2040-9. 